\title{
Les abeilles du genre Colletes (Hymenoptera, Colletidae) en Presqu'île guérandaise (Loire-Atlantique, France)
}

Par Gilles MAHÉ *

\begin{abstract}
At least seven species of Colletes have been found in the Guérande Peninsula. This paper illustrates the habitats where the species have been found, as well as the flowering plants visited.
\end{abstract}

Résumé. Au moins sept espèces de Colletes sont présentes en Presqu'île guérandaise. Cet article indique dans quels milieux et sur quelles plantes l'auteur a observé ces abeilles.

Mots-clés. Colletes, plantes butinées.

\section{Introduction}

Certaines abeilles du genre Colletes peuvent former des agrégats de très nombreux nids. Sur les dunes de Pont-Mahé (Loire-Atlantique, Assérac; WGS84 $\left.47,4468^{\circ} \mathrm{N} / 2,4545^{\circ} \mathrm{W}\right)$ j’ai observé des dizaines de milliers de nids de collète du saule, Colletes cunicularius (L.), avec une densité pouvant atteindre la centaine de nids au mètre carré. Fin mars, lors de belles journées ensoleillées des milliers d'abeilles virevoltent au ras du sol. Il est amusant de voir les badauds inquiets s'interroger en restant à bonne distance. Quand on commence à s'intéresser aux abeilles sauvages, on fait généralement assez rapidement la connaissance des collètes. Je n'ai pas échappé à cette règle et je rapporte ici quelques observations faites en Presqu'île guérandaise entre 2000 et 2008. Le territoire de ladite presqu'île (Figure 1) s'étend entre l'océan et les marais de Grande Brière qui communiquaient autrefois largement avec l'estuaire de la Loire. La présence de cordons dunaires et de terrains plus ou moins sablonneux convient bien à ces abeilles qui trouvent là des sites favorables pour le creusement de leur terrier.

Les abeilles du genre Colletes sont restées longtemps mal étudiées. Un travail conséquent a été réalisé par Noskiewicz (1936). Plus récemment Janvier (1980) a décrit de façon très détaillée la structure et la composition des nids de 25 espèces. Les collètes ont longtemps été considérées comme des espèces primitives mais les études génétiques récentes (Danforth et al. 2006) les placent désormais parmi les abeilles les plus récentes dans l'arbre évolutif des abeilles (voir à ce sujet l'article de Michez (2007)). Aujourd'hui, universitaires et amateurs se passionnent pour les collètes. Les préférences alimentaires strictes de ces abeilles mais aussi les relations étonnantes qui les lient à d'autres espèces en font l'objet d'études diverses comme celles menées par Vereecken et al. (2007) sur les phéromones sexuelles.

* Gilles Mahé, Rue de la matte 18, F-44600 Saint-Nazaire, France. E-mail: gilles.mahe@wanadoo.fr

\section{Colletes cunicularius (L.) (Collète du saule)}

Cette grosse abeille (jusqu'à $17 \mathrm{~mm}$ ) est commune en Loire-Atlantique. Elle affectionne les sites qui lui offrent à la fois le gîte (zone sablonneuse) et le couvert avec principalement les saules (Salix sp., Salicaceae). C'est la seule espèce du genre Colletes à voler en mars-avril. Elle se trouve principalement dans les milieux dunaires du bord de mer, mais elle est également présente dans l'intérieur des terres (anciennes carrières de sable), sur des remblais sablonneux (en bord de Loire) et même dans des cimetières. Ces abeilles forment généralement des bourgades très populeuses (Figure 2). On y rencontre régulièrement Sphecodes albilabris (FABRICIUS) (Halictidae), abeille parasite qui est facilement repérable par sa taille (jusqu'à $14 \mathrm{~mm}$ ) et son abdomen rouge.

Bien que $C$. cunicularius ait une préférence pour les fleurs du saule (Figure 3), je l'ai souvent observée en Loire-Atlantique récoltant assidûment le pollen d'autres plantes telles que l'ajonc d'Europe (Ulex europaeus L.), l'alaterne (Rhamnus alaternus L.) ou le prunellier (Prunus spinosa L.) (Figures 4 à 6). En examinant des charges de pollen au microscope j'ai vérifié que parfois elles ne ramenaient au nid que du pollen d'ajonc. Les collètes que j'ai capturées sur la dune de Bonne-source (LoireAtlantique, Pornichet; WGS84 47,2518 ${ }^{\circ} \mathrm{N} / 2,3283^{\circ} \mathrm{W}$ ) n'étaient elles chargées que de pollen d'alaterne. Les saules étant absents à proximité, il serait intéressant de vérifier si cette abeille peut se passer complètement des saules au cours de sa vie. (voir aussi Bischoff et al. (2003), Müller \& Kuhlmann (2008) et l'article de Vanderplanck et al. (2009) dans ce numéro).

\section{Colletes hederae SCHMIDT \& WESTRIGH (Collète du lierre)}

C'est assurément l'espèce du genre Colletes la plus répandue en Presqu'île guérandaise. Le peu de localités signalées sur la carte (Figure 1) vient simplement du fait qu'elle est si commune que je ne prends pas la peine de la noter quand je la rencontre. On la trouve à partir de 


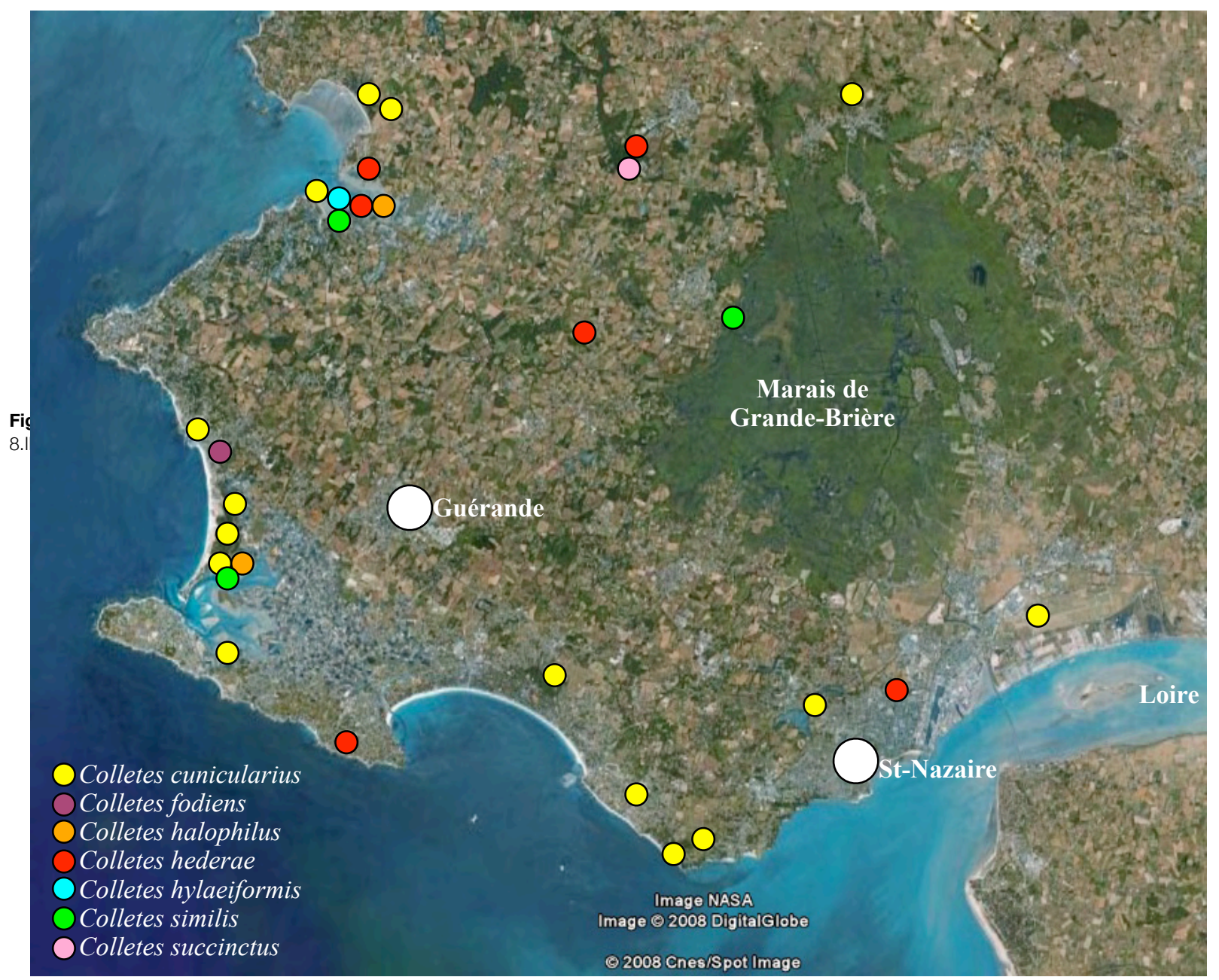

Figure 1. Quelques sites à abeilles du genre Colletes en Presqu'île Guérandaise (Loire-Atlantique, France)

début septembre à peu près partout où il y a de beaux massifs de lierres (Hedera helix L.) en fleurs. Pour faire son nid, elle s'accommode de petits espaces bien exposés, de préférence à végétation rase ou espacée (talus, chemin, pelouse, etc.). Les femelles butinent principalement le pollen de lierre mais en bord de mer je les ai souvent observées qui récoltaient le pollen de baccharis (Baccharis halimifolia, L.) (Figure 7). Les mâles butinent le nectar de lierre et d'autres plantes. Je les ai notés sur la callune (Calluna vulgaris L.), le sarrasin (Fagopyrum esculentum MOENCH) (Figure 8) et d'autres polygonacées (Polygonum L. sp.). Sur les sites parcourus en presqu'île guérandaise, j'ai souvent rencontré les

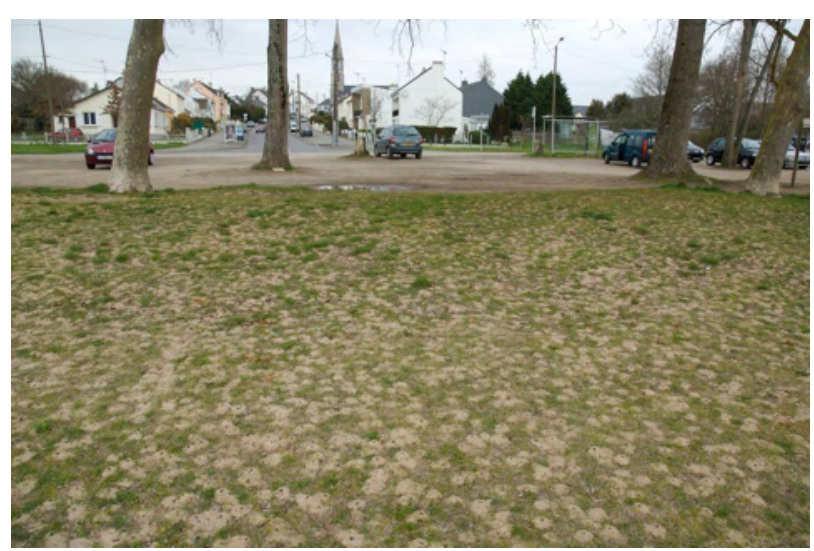

Figure 2. Concentration de nids de Colletes cunicularius L. (Photo G Mahé, St-Nazaire (F-44)) abeilles-coucou Epeolus cruciger (PANZER) et E. variegatus (L.), probables parasites des collètes du lierre. J'ai également mentionné la présence de Stenoria analis (SCHAUM) (Coleoptera, Meloidae), parasite avéré de cette abeille, en plusieurs points de la presqu'île (Mahé 2007; Vereecken \& Mahé 2007).

\section{Colletes succinctus $\mathrm{L}$. (Collète de la callune)}

Colletes succinctus se maintient dans le département mais semble assez discrète. Je n'ai trouvé aucun agrégat de centaines de nids comme peuvent former les collètes du lierre. En Presqu'île guérandaise je l'ai cherchée

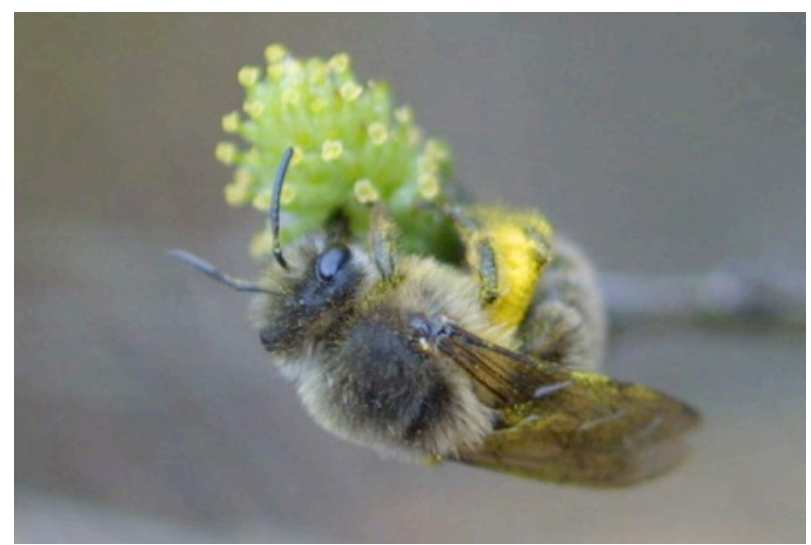

Figure 3. Femelle de Colletes cunicularius sur inflorescence de saule (Salix sp, Salicaceae) (Photo G Mahé) 

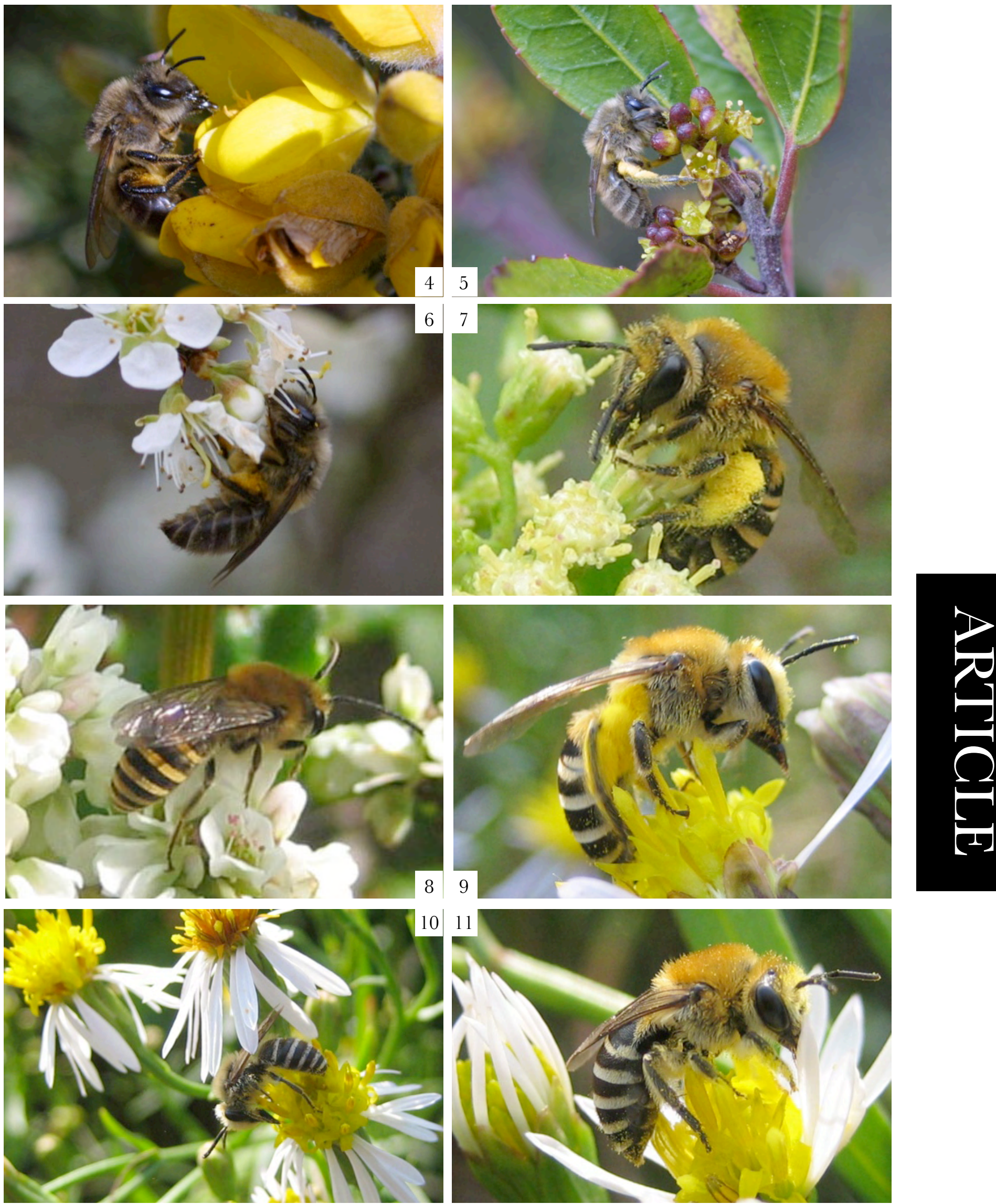

Figures 4 à 11. Quelques observations des abeilles du genre Colletes sur leurs plantes-hotes en Loire-Atlantique. 4. Femelle de C. cunicularius sur Ulex europaeus (Fabaceae); 5. Femelle de C. cunicularius sur Rhamnus alaternus (Rhamnaceae); 6. Femelle de C. cunicularius sur Prunus sp. (Rosaceae); 7. Femelle de C. hederae sur Baccharis halimifolia L. (Asteraceae); 8. Mâle de C. hederae sur Fagopyrum esculentum (Polygonaceae); 9. Femelle de C. halophilus sur Aster tripolium (Asteraceae); 10. Mâle de C. halophilus sur Aster tripolium (Asteraceae); 11. Femelle de C. similis sur Aster tripolium (Asteraceae) (Photos G. Mahé). 


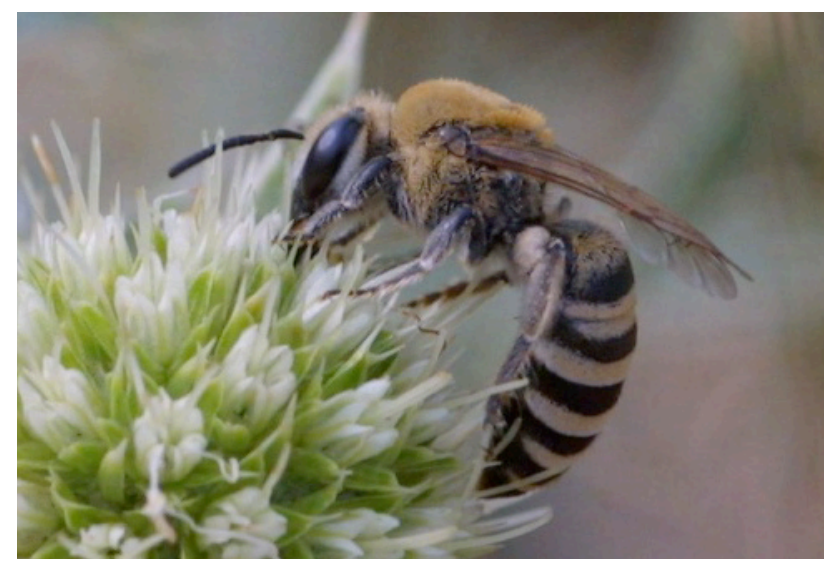

Figure 12. Femelle de Colletes hylaeiformis sur Eryngium campestre (Apiaceae) (Photo G Mahé, Mesquer (F-44))

longtemps avant d'en capturer quelques exemplaires fin août 2006 sur la callune dans le bois de la cour aux loups (Loire-Atlantique, Herbignac; WGS84 $\left.47,4338^{\circ} \mathrm{N} / 2,3356^{\circ} \mathrm{W}\right)$.

\section{Colletes halophilus VERHOEFF (Collète des asters)}

Cette espèce semble strictement inféodée aux vasières et prés salés à Aster tripolium (L.) qui est sa plante favorite (Figures 9 et 10). Je l'ai quelques fois capturée sur les Baccharis. C'est une espèce rare à l'échelle mondiale. Elle n'est connue que des côtes de France, Belgique, Pays-Bas et Royaume-Uni (Genoud \& Dittlo 2007). En Presqu'île guérandaise, de petites colonies se trouvent dans le traict de Rostu (Loire-Atlantique, Mesquer; WGS84 $47,4142^{\circ} \mathrm{N} / 2,4526^{\circ} \mathrm{W}$ ), et dans le petit traict de Pen-Bron (Loire-Atlantique, La Turballe; WGS84 47,3122 $\left.{ }^{\circ} \mathrm{N} / 2,5005^{\circ} \mathrm{W}\right)$.

\section{Colletes similis SGHENGK}

Cette espèce ressemble à $C$. halophilus mais lorsque les deux espèces sont présentes en même temps on la distingue par sa taille plus petite et son corps plus trapu (Figure 11). Elle a une préférence pour les astéracées mais on la trouve aussi sur d'autres plantes. Je l'ai capturée sur Aster tripolium (L.), Pulicaria dysenterica (L.), Leucanthemum vulgare LAM., Achillea millefolium (L.) et Foeniculum vulgare MILLER. Sa moins grande exigence alimentaire, comparée à d'autres collètes, fait que cette espèce n'est pour l'instant pas menacée en LoireAtlantique.

\section{Colletes hylaeiformis EVERSMANN (Collète de l'Eryngium)}

Cette très belle espèce (Figures 12 et 13), dont la femelle se distingue des autres collètes par la pilosité rase de son thorax, est spécialisée sur les Eryngium. Elle semble rare en Loire-Atlantique. Je ne l'ai observée qu'une seule fois sur Eryngium campestre L. (Apiaceae) fin juillet 2006 sur la Baule de Merquel (Loire-Atlantique, Mesquer WGS84 47,4130 ${ }^{\circ} \mathrm{N} / 2,4645^{\circ} \mathrm{W}$ ).

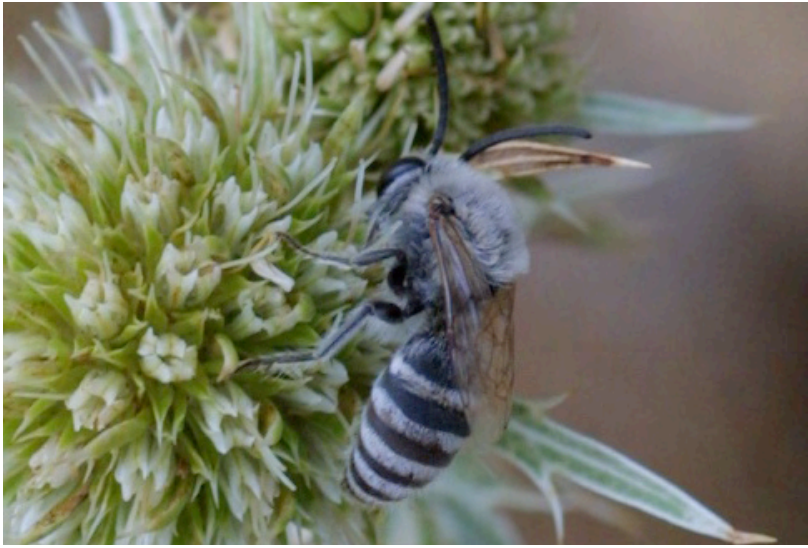

Figure 13. Mâle de Colletes hylaeiformis sur Eryngium campestre (Apiaceae) (Photo G Mahé, Mesquer (F-44))

\section{Colletes fodiens (GEOFFROY)}

J'avais déjà capturé cette espèce sur l'immortelle des dunes, Helichrysum stoechas (L.), en Presqu'île de Crozon (Finistère, Camaret; WGS84 48,2623ํN/4,6128 ${ }^{\circ} \mathrm{W}$ ). C'est sur cette même plante que je l'ai trouvée en Presqu'île guérandaise en août 2000 sur la dune de Belmont (Loire-Atlantique, La Turballe; WGS84 $\left.47,3592^{\circ} \mathrm{N} / 2,5186^{\circ} \mathrm{W}\right)$. Elle semble préférer les astéracées. Je l'ai notée qui butinait Senecio jacobaea (L.) mais je l'ai aussi capturé sur l'apiacée Eryngium campestre (L.).

\section{Colletes daviesanus SMITH}

Je n'ai pas encore trouvé cette espèce en Presqu'île guérandaise. Elle est spécialisée sur les astéracées, et assez commune en France et en Europe d'après Janvier (1980) qui la signale en Bretagne. C'est donc une espèce potentiellement présente à rechercher.

\section{Colletes marginatus SMITH}

Cette espèce réputée spécialisée sur les Fabacées mais observée également sur les Apiacées et les Astéracées est signalée principalement sur les sites côtiers de France, Belgique, Pays-Bas, Allemagne et Royaume-Uni (Devalez \& Vereecken 2008). Je l'ai observée en 2000 et 2001 en Bretagne à Camaret (Finistère; WGS84 48,2623 ${ }^{\circ} \mathrm{N} / 4,6128^{\circ} \mathrm{W}$ ), Crozon (Finistère; WGS84 $48,2331^{\circ} \mathrm{N} / 4,4375^{\circ} \mathrm{W}$ ) et le Conquet (Finistère; WGS84 48,3703 ${ }^{\circ} \mathrm{N} / 4,7595^{\circ} \mathrm{W}$ ) dans des milieux arrière-dunaires à chaque fois sur du fenouil (Foeniculum vulgare MILLER, Apiaceae). Cette abeille pourrait être présente en Presqu'île guérandaise dans des milieux similaires. Il serait intéressant de l'y rechercher.

\section{Menaces sur les habitats}

Les collètes ayant des exigences alimentaires assez strictes, leur survie dépend de la conservation des habitats où se trouvent en relative abondance les plantes dont elles ont besoin. Ainsi, la régression considérable des landes à callunes (Calluna vulgaris L.) en Loire- 


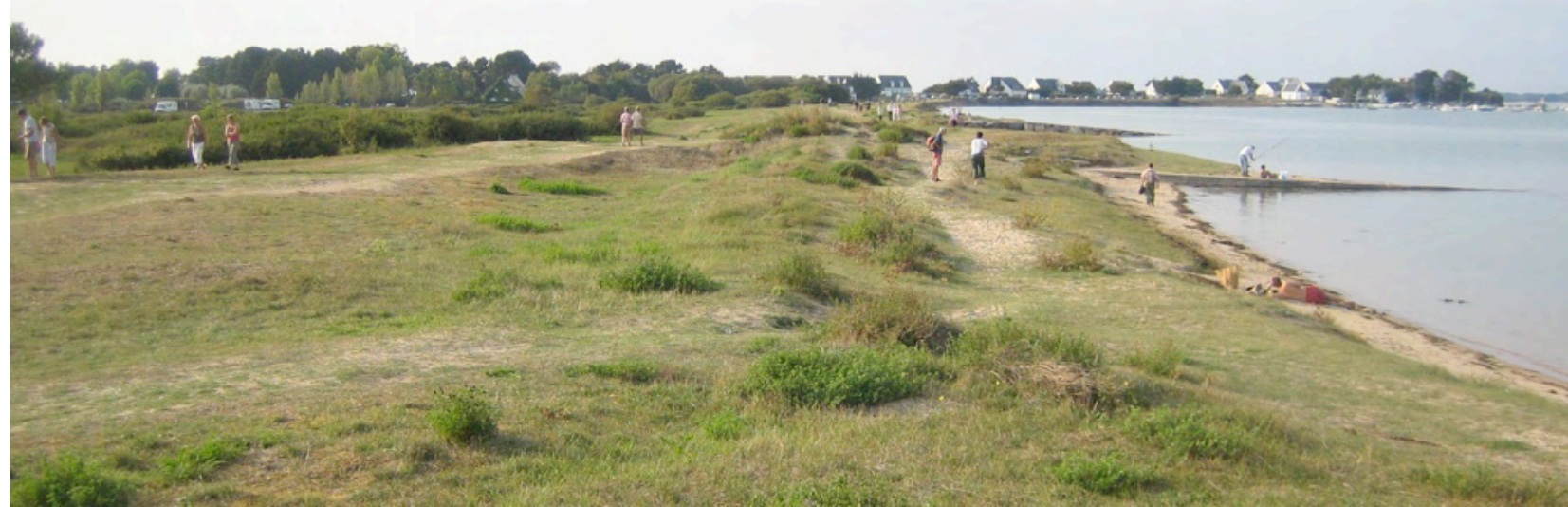

Figure 14. La Baule de Merquel, Mesquer (Photo G Mahé)

Atlantique au cours du dernier siècle explique en grande partie la raréfaction de la collète de la callune (C. succinctus) dans le département. Les espaces dunaires tels que la Baule de Merquel à Mesquer où la dune de Belmont à La Turballe sont des sites remarquables qui offrent aux apoïdes d'une part une flore diversifiée dont la floraison s'étale de mars à octobre, et d'autre part de bonnes possibilités de nidification. C'est sur ces deux sites que j’ai signalé pour la première fois en LoireAtlantique la présence de l'Ophrys de la passion, Ophrys passionis Sennen (Mahé 2001). Cette orchidée n'a qu'un seul pollinisateur connu : l'abeille des sables Andrena pilipes FABRICIUS qui est commune sur les deux sites.

Le cordon sableux de La Baule de Merquel (Figure 14), classé en espace naturel sensible par le département, abrite cinq espèces de collètes, et au moins 60 autres espèces d'abeilles sauvages y ont été identifiées. C'est un site agréable très fréquenté par les promeneurs. Se pose donc la question de la gestion du milieu et de sa fréquentation. En 2008 d'importants travaux y ont été réalisés pour d'une part éradiquer les baccharis et d'autre part canaliser les personnes. Il sera intéressant d'évaluer l'impact des aménagements sur la faune des abeilles sauvages d'ici à quelques années. La dune de Belmont est une dune grise en très bon état de conservation, ce qui est exceptionnel pour un espace péri-urbain. La clôture des parcelles privées par les propriétaires a eu pour effet d'y limiter le piétinement. Cette dune est d'une très grande richesse pour sa flore et les apoïdes qu'elle héberge. J'ai signalé en 2006 la présence de l'abeille méridionale Anthophora mucida GRIBODO, qui se trouve là en limite nord de sa répartition. Malheureusement cet espace de quelques hectares est en grande partie constructible et se réduit régulièrement par la construction de nouvelles habitations.

\section{Conclusion}

Avec au moins sept espèces d'abeilles du genre Colletes, plus quelques autres potentiellement présentes, la Presqu'île guérandaise est un territoire qui comporte des milieux favorables pour ces abeilles solitaires assez exigeantes aussi bien du point de vue des ressources alimentaires que des zones de nidification. A titre de comparaison, c'est l'équivalent de la faune des collètes de Belgique. Mais ces milieux sont extrêmement fragiles et menacés par l'extension de l'urbanisation et des infrastructures touristiques. Il conviendrait donc de conserver et de classer en zone naturelle l'ensemble des petits espaces dunaires aujourd'hui en grande partie morcelés et disséminés en Presqu'île guérandaise entre Saint-Nazaire et Pénestin.

\section{Références bibliographiques}

Bischoff I, Feltgen K \& Breckner D, 2003. Foraging strategy and pollen preferences in Andrena vaga (PANZER) (Hym. Andrenidae) and Colletes cunicularius (L.) (Hym. Colletidae). Fournal of Hymenoptera Research 12 (2): 220-237

Danforth BN, Sipes SD, Fang J \& Brady SG, 2006. The history of early bee diversification based on five genes plus morphology. Proceedings of the National Academy of Sciences of the USA 103: 15118-15123.

Devalez J \& Vereecken NJ, 2008. Nouvelles données sur la présence de Colletes marginatus SMITH (Hymenoptera, Colletidae) sur le littoral belge. OSMIA 2: 3-4.

Janvier H, 1980. Comportements d'abeilles Colletidae Hymenoptera). Mémoire original diffusé par l'auteur, 344 pp.

Mahé G, 2001. L'Ophrys de la passion, Ophrys passionis SENNEN ex J.P. DEVILLERS-Terschuren, 1994, en Loire-Atlantique et en Vendée. Le Naturaliste vendéen 1: 41-42.

Mahé G, 2007. Observations en Loire-Atlantique (France) de Stenoria analis (SCHAUM) (Coleoptera, Meloidae), cleptoparasite de Colletes hederae SCHMIDT \& WESTRICH (Hymenoptera, Colletidae). OSMIA 2: 11-15

Michez D, 2007. La nouvelle classification des abeilles (Hymenoptera, Apoidea, Apiformes) ou la chute de l'abeille mellifère (Apis mellifera L.) de son piédestal. OSMIA 1: 23-26.

Müller A \& Kuhlmann M, 2008. Pollen hosts of western palaearctic bees of the genus Colletes (Hymenoptera: Colletidae): the Asteraceae paradox. Biological fournal of the Linnean Society 95: 719-733.

Noskiewicz J, 1936. Die palaearktischen Colletes-Arten. Prace nankowe; wydawnictwo Towarzystwa naukowego we Lwowie 3: 1-532.

Vereecken NJ \& Mahé G, 2007. Larval aggregations of the blister beetle Stenoria analis (ScHAUM) (Coleoptera: Meloidae) sexually deceive patrolling males of their host, the solitary bee Colletes hederae SCHMIDT \& WESTRICH (Hymenoptera: Colletidae). Annales de la Société Entomologique de France 43(4) : 493-496.

Vereecken NJ, Mant J \& Schiestl FP, 2007. Population differentiation in female mating signals and male preferences in a solitary bee. Behavioural Ecology and Sociobiology 61(5): 811-821. 